\title{
Mechanical characterization of microparticles by scattered ultrasound
}

Ronald A. Roy, and Robert E. Apfel

Citation: The Journal of the Acoustical Society of America 87, 2332 (1990); doi: 10.1121/1.399079

View online: https://doi.org/10.1121/1.399079

View Table of Contents: https://asa.scitation.org/toc/jas/87/6

Published by the Acoustical Society of America

\section{ARTICLES YOU MAY BE INTERESTED IN}

Acoustic radiation force on a compressible cylinder in a standing wave

The Journal of the Acoustical Society of America 116, 201 (2004); https://doi.org/10.1121/1.1753291

Acoustic-Radiation Force on a Solid Elastic Sphere

The Journal of the Acoustical Society of America 46, 1139 (1969); https://doi.org/10.1121/1.1911832

Extension of acoustic levitation to include the study of micron-size particles in a more compressible host liquid The Journal of the Acoustical Society of America 71, 1261 (1982); https://doi.org/10.1121/1.387776

Acoustic radiation force on a spherical particle in a viscous heat-conducting fluid. I. General formula The Journal of the Acoustical Society of America 101, 713 (1997); https://doi.org/10.1121/1.418035

Acoustic radiation force on a spherical particle in a viscous heat-conducting fluid. III. Force on a liquid drop The Journal of the Acoustical Society of America 101, 731 (1997); https://doi.org/10.1121/1.417961

Efficient finite element modeling of radiation forces on elastic particles of arbitrary size and geometry The Journal of the Acoustical Society of America 133, 1885 (2013); https://doi.org/10.1121/1.4794393 


\title{
Mechanical characterization of microparticles by scattered ultrasound
}

\author{
Ronald A. Roya) and RobertE. Apfel \\ Department of Mechanical Engineering, Yale University, Box 2159 Yale Station, New Haven, Connecticut \\ 06520
}

(Received 23 April 1989; accepted for publication 11 January 1990)

\begin{abstract}
A technique for determining the compressibility and density of individual microparticles in suspension is described. The particles have diameters on the order of $10 \mu \mathrm{m}$. Ultrasonic tone bursts of $2-\mu$ s duration and $30-\mathrm{MHz}$ center frequency scatter from individual particles as they traverse the confocal zone of two transducers. The resulting scattered tone bursts are detected at $90^{\circ}$ and $180^{\circ}$ (backscattering). The received rf signals are demodulated, peak detected, digitized, and stored in computer memory. Using Rayleigh scattering theory, the compressibility and density of a particle can be computed given knowledge of the particle size and host fluid properties. Results of experiments with latex microspheres are presented and compared with calculations based on long-wavelength (Rayleigh) and elastic scattering theory.
\end{abstract}

PACS numbers: 43.20.Fn, 43.35.Bf, 43.35.Yb

\section{INTRODUCTION}

The task of performing rapid and quantitative microparticle analysis has attracted considerable attention in a number of areas. Many industrial applications, such as multiphase flow monitoring and quality control, are well suited to microparticle detection techniques. Viability of very pure processes can be established through detectors that are sensitive to particulate contamination or precipitation. In cases where either liquid or solid particle formation is part of the manufacturing process, particle analyzers can yield realtime information useful to the control process, provide a mechanism for quality control, or play a role in product evaluation.

Biological applications for cell characterization and separation technologies are numerous because changes in optical and/or mechanical properties of cells may indicate disease. Researchers are often faced with the difficult task of resolving mixed cell populations into distinct homogeneous populations. Automated cell analyzers and sorters can process large numbers of cells; this facilitates the harvesting of large quantities of pure populations and permits one to monitor or identify very small subpopulations within a heterogeneous parent distribution.

In this paper, we describe a procedure that is amenable to in vitro cell characterization. In the context of our work, "microparticle characterization" refers to the determination of the volume, density, and adiabatic compressibility of uniform, spherical particles in suspension. Suspended microparticle characterization techniques can be subdivided into two classes: multiple-particle and single-particle characterizers.

Multiple-particle techniques determine mean properties averaged over an ensemble of particles. Ensemble size mea-

\footnotetext{
") Current address: National Center for Physical Acoustics, P.O. Box 847, University, Mississippi 38677.
}

surements can be performed by measuring the density of a suspension of a known number of particles of known density, or by using optical techniques such as photon correlation spectroscopy. ${ }^{1}$ Density-gradient centrifugation provides information regarding the mean density of a particle population and facilitates the separation of the various constituents of a mixed population. ${ }^{2}$ Measurements of the bulk elastic properties of microparticles is limited primarily to acoustic techniques such as suspension sound velocity measurements ${ }^{3}$ and acoustic levitation. ${ }^{4}$

Single-particle procedures offer the advantage of being able to establish distributions of properties for a population of particles. In some cases, two or more measurement schemes can be simultaneously employed, resulting in multiparameter characterization. ${ }^{5}$ There are some disadvantages, however. Single-particle techniques tend to be expensive and are susceptible to signal-to-noise limitations. Also, depending on the application, particle handling may prove problematic. Individual particle sizing can be accomplished optically via microscopy or forward laser scattering. ${ }^{6}$ Electrozone sensing instruments ${ }^{7}$ provide a convenient technique for sizing and counting large numbers of particles. Neither the optical nor impedance technique are sensitive to mechanical properties. Indeed, all of the commonly used procedures for measuring density and compressibility are multiple-particle techniques.

We have developed an acoustic scattering technique for quantitatively determining the density and adiabatic compressibility of large numbers of individual microparticles in suspension. Relevant particle sizes, which for our work range from $4 \mu \mathrm{m}$ to about $15 \mu \mathrm{m}$ in diameter, can be adjusted by proper scaling of the acoustic frequency. Narrow-band, ultrasonic tone bursts scattered from individual particles are detected at two angles. Using either long-wavelength or weak-scattering theory, we can calculate the mechanical properties of an individual particle, provided we possess $a$ priori knowledge of the particle size. We can subsequently 
compute mean properties for a homogeneous population or determine properties for subpopulations within a heterogeneous distribution.

The apparatus described herein is a modification of a system developed by Roos. ${ }^{8,9}$ The purpose of this paper is to describe the technique and the underlying theory. We present results of experiments performed on polystyrene microspheres, which we use as calibration particles. Measurements made (using a weak-scattering approximation) on biological particles such as Chinese hamster ovary cells and human granulocytes will be presented in a later paper.

\section{SCATTERING FROM SPHERICAL PARTICLES: AN OVERVIEW}

When a sound wave traveling through a homogeneous medium encounters a local inhomogeneity, a portion of the wave is scattered in all directions. The resulting wave is the superposition of the incident and scattered waves. The nature of the scattered field depends on the acoustical properties of the scatterer and the host material, the acoustic frequency, and the size and shape of the scatterer. In the most general case, the host and the scatterer would admit the propagation of both compressional and shear waves, the relevant boundary conditions being the continuity of normal stress, normal velocity, tangential stress, and tangential velocity across the surface of the scatterer. For our purposes, we shall consider the scattering from both fluid and elastic spheres suspended in a fluid host. Thus the tangential components of the velocity and stress fields vanish.

\section{A. Scattering from a fluid sphere in a fluid host}

We consider first the case of harmonic plane waves of amplitude $P_{0}$ and angular frequency $\omega$ propagating through an infinite, inviscid fluid of density $\rho$ and compressibility $\kappa$. These waves are incident upon a homogeneous, inviscid fluid sphere of radius $a$, density $\rho^{\prime}$, and compressibility $\kappa^{\prime}$. The stress fields outside and inside the scatterer satisfy linear compressional wave equations, where the relevant boundary conditions are the continuity of normal stress and normal velocity across the interface. Using eigenfunction expansions, Anderson ${ }^{10}$ obtained an exact solution for the scattered wave. The farfield scattered pressure amplitude $P_{s}$ is given by

$$
P_{s}(r)=\left[\left(P_{0} / r\right) e^{\mathrm{ikr}}\right] \Phi, \text { for } r \gg a,
$$

where

$$
\Phi=\frac{i}{k} \sum_{m=0}^{\infty}(-1)^{m}(2 m+1) \frac{P_{m}(\cos \theta)}{\left(1+i C_{m}\right)} .
$$

In this expression, $r$ is the radial distance from the center of the scatterer to the field point, $k$ is the wavenumber in the host fluid, the $P_{m}$ are the Legendre polynomials, and $\theta$ is the scattering angle ( $\theta=180^{\circ}$ for backscattering). The coefficients $C_{m}$, which result from matching the boundary conditions, are given in Ref. 10. Note that $P_{s}$ takes on the form of a spherical wave modulated by an angular distribution function $\Phi$, which is a function of $\omega, \theta, a$, and the scatterer and host properties. We shall also refer to $\Phi$ as the "scattering strength."

\section{B. Scattering from an elastic sphere in a fluid host}

Suppose now that the scatterer is a uniform elastic sphere. A straightforward extension of the normal mode expansion technique yields the solution to the elastic scattering problem. One defines compressional and shear wave velocities $\left(c^{\prime}, c_{s}^{\prime}\right)$ in terms of the Lamé constants $\left(\lambda^{\prime}, \mu^{\prime}\right)$ for the solid obstacle. These are given by

$$
c_{2}^{\prime}=\left(\lambda^{\prime}+2 \mu^{\prime}\right) / \rho^{\prime}, \quad c_{s}^{\prime 2}=\mu^{\prime} / \rho^{\prime} .
$$

The displacement in the scatterer is the sum of both compressional and shear wave displacements. Two independent wave equations result: scalar and vector velocity potential equations for the compressional and shear wave components, respectively. Add a third boundary condition, the vanishing of the tangential stress at the surface of the scatterer, and the subsequent calculations are completely analogous to the fluid case (the reader may choose to refer to the excellent articles by Faran, ' Hickling, ${ }^{12}$ and Hay and Burling ${ }^{13}$ for further details). The resulting expression for the farfield scattering strength is ${ }^{13}$

$$
\Phi=\frac{i}{k} \sum_{m=0}^{\infty}(2 m+1) \sin \left(\eta_{m}\right) \exp \left(-i \eta_{m}\right) P_{m}(\cos \theta)
$$

An expression for the phase angle for the $m$ th normal mode $\eta_{m}$ is given in Ref. 13.

\section{The long-wavelength approximation}

Equations (2) and (4) are applicable solutions to the direct scattering problem. However, the task of inverting $\Phi$ to yield the scatterer properties given knowledge of the host properties and the incident and scattered fields must be carried out numerically, an approach that entails substantial computational overhead. We require a readily computable solution to the inverse scattering problem, thereby facilitating real-time data analysis. Consider, therefore, the case where the radius of the fluid scatterer is much less than the wavelength of sound in the host. To lowest order in $k a$, only the $m=0$ and 1 terms in the expansion are retained and Eq. (2) reduces to

$$
\Phi=(1 / 4 \pi)\left(k^{2} V\right)(\delta \kappa+\delta \rho \cos \theta),
$$

where $V$ is the scatterer volume. The functions $\delta \kappa$ and $\delta \rho$ are the compressibility and density contrasts given by

$\delta \kappa \equiv\left(\kappa^{\prime}-\kappa\right) / \kappa$, and $\delta \rho \equiv 3\left(\rho^{\prime}-\rho\right) /\left(2 \rho^{\prime}+\rho\right)$.

Equation (5) is the Rayleigh result for long-wavelength scattering from fluid spheres. ${ }^{14}$ The monopole term depends on the compressibility contrast and corresponds to a "breathing mode" oscillation, in which the sphere pulsates radially. The dipole term, which depends on the density contrast, results from the motion of the center of mass of the scatterers along the axis of the incident wave.

A similar argument can be made for the elastic scatterer. To lowest order in $k a$, Eq. (4) also reduces to Eqs. (5), where the bulk compressibility of the solid is given in terms of its Lamé constants by

$$
\kappa^{\prime}=\left(\lambda^{\prime}+2 \mu^{\prime} / 3\right)^{-1} .
$$

In the Rayleigh limit, the particle is too small to support any 
of the shear wave resonance modes. Thus an elastic sphere can be modeled by a fluid sphere with an effective compressibility given by Eq. (6).

Inverting Eqs. (5) to obtain the scatterer properties is straightforward. Let $\Phi_{90}$ and $\Phi_{180}$ be the scattering strengths measured at $\theta=90^{\circ}$ and $180^{\circ}$, respectively. From Eq. 5(a) we solve for the contrasts:

$$
\delta \kappa=4 \pi \Phi_{90} /\left(k^{2} V\right)
$$

and

$$
\delta \rho=4 \pi\left(\Phi_{90}-\Phi_{180}\right) /\left(k^{2} V\right),
$$

which can be combined with Eq. ( $5 b)$ to yield the particle properties:

$\kappa^{\prime}=\kappa(1+\delta \kappa)$, and $\rho^{\prime}=\rho[(3+\delta \rho) /(3-2 \delta \rho)]$.

Thus, in the long-wavelength limit, we can determine the compressibility and density of the scatterer by measuring the scattered signal at $90^{\circ}$ and $180^{\circ}$ (or any two angles for that matter) provided we possess a priori knowledge of the host compressibility and density as well as the scatterer size.

\section{EXPERIMENTAL APPARATUS}

Figure 1 is a block diagram of the apparatus used to measure scattering strengths. Individual particles are convected through the confocal region of two submerged transducers by a coaxial jet flow. High-frequency, narrow-band tone bursts that scatter from a particle are detected at $180^{\circ}$ (channel A) and $90^{\circ}$ (channel B). The received echoes are demodulated, peak detected, digitized, and stored in computer memory for subsequent processing. The traversal of a single particle (particle event) results in several scattered echoes (scattering event). The objective is to determine the particle scattering strength, which is the peak echo amplitude produced by the particle event.

\section{A. Transducers and particle handling}

We use matched, unbacked transducers that incorporate a $1.5-\mathrm{cm}$-diam, $3.0-\mathrm{cm}$ focal length, machined styrene lens bonded to a $0.95-\mathrm{cm}$-diam lithium niobate crystal. The 3-dB beamwidth is about $275 \mu \mathrm{m}$ (measured in the focal plane using a $10-\mu \mathrm{m}$-diam cylindrical target fashioned from a quartz filament). The sidelobes are approximately $15 \mathrm{~dB}$ down from the central maxima. We choose a $30-\mathrm{MHz}$ resonance frequency in order to maximize acoustic sensitivity without severely compromising the long-wavelength scattering assumption for biological particles up to $10 \mu \mathrm{m}$ in diameter. ${ }^{15}$ (The value of $k a$ above which the approximation fails depends on the material properties of the scatterer and the host. For example, the long-wavelength assumption fails at low $k a$ for high-contrast scatterers.) Narrow-band signals are desirable since they facilitate broadband noise rejection. A 2- $\mu$ s-long tone burst yields a $500-\mathrm{kHz}$ signal bandwidth centered at $30 \mathrm{MHz}$.

Figure 2 illustrates the mechanical system for positioning the transducers and the jet flow assembly. Each transducer is held in place by a Plexiglas support arm that is affixed to an $X-Y-Z$ micropositioning stage. A third $X-Y-Z$ micropositioner is used to orient the jet assembly so that the particle flow passes through the confocal region of the two

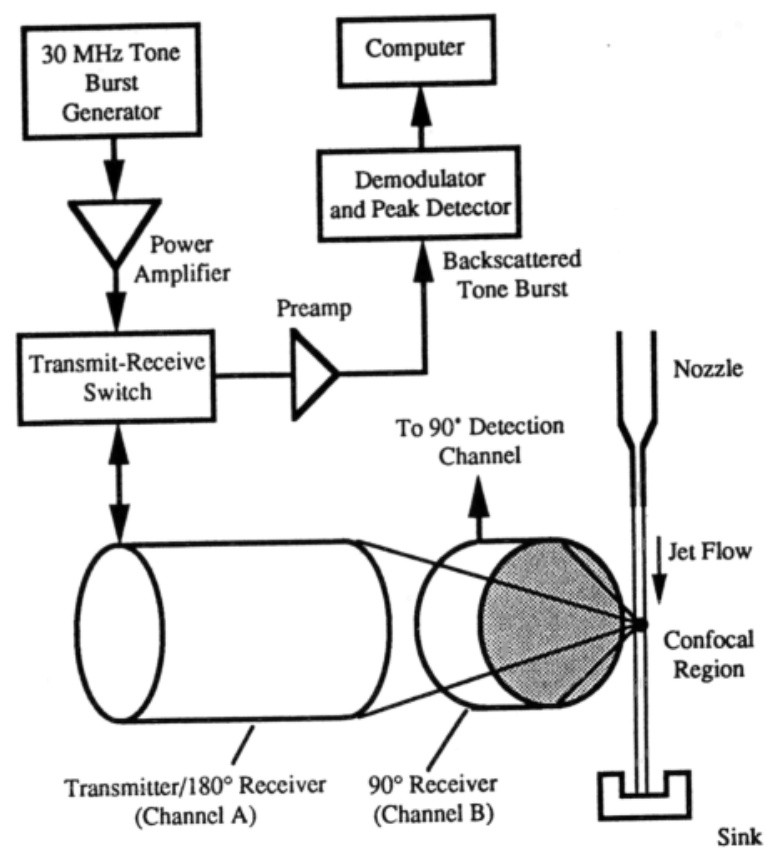

FIG. 1. Block diagram of the microparticle characterization apparatus ( $180^{\circ}$ detection channel only).
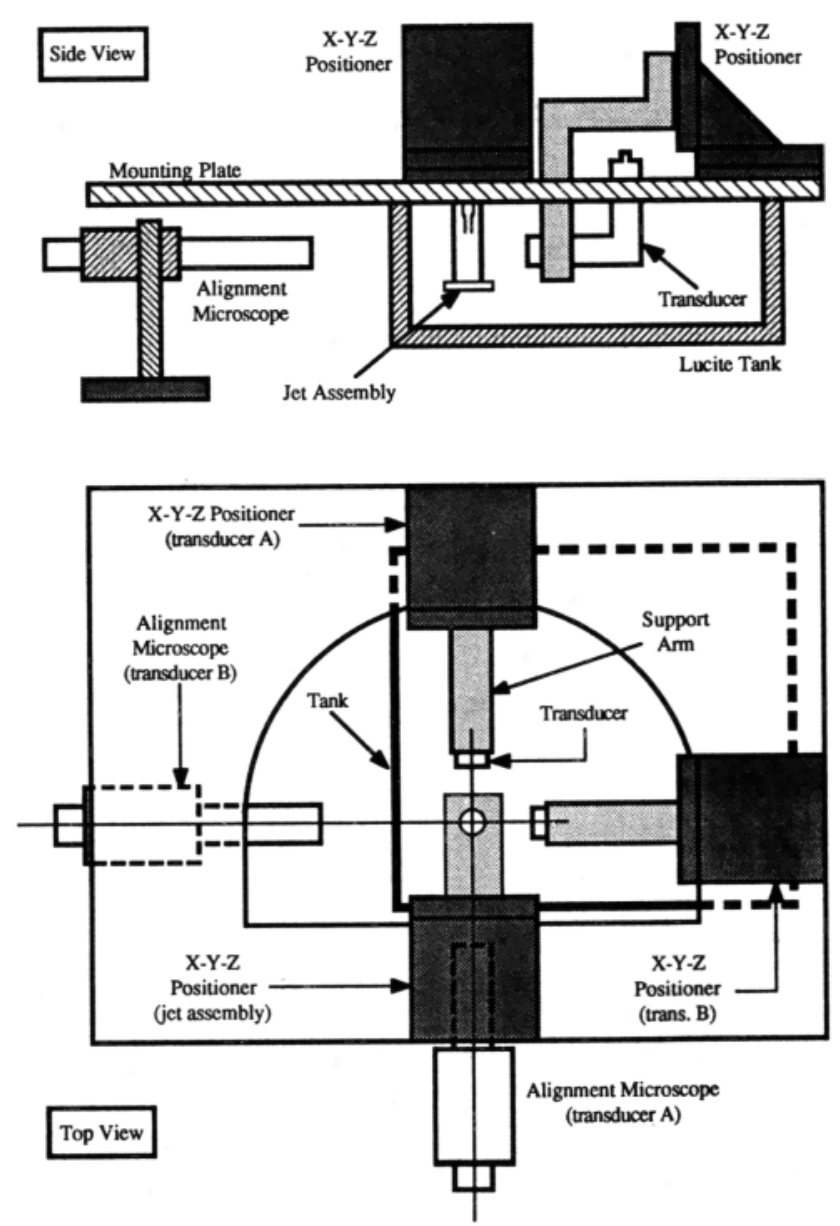

FIG. 2. Transducer mounting and alignment system. 


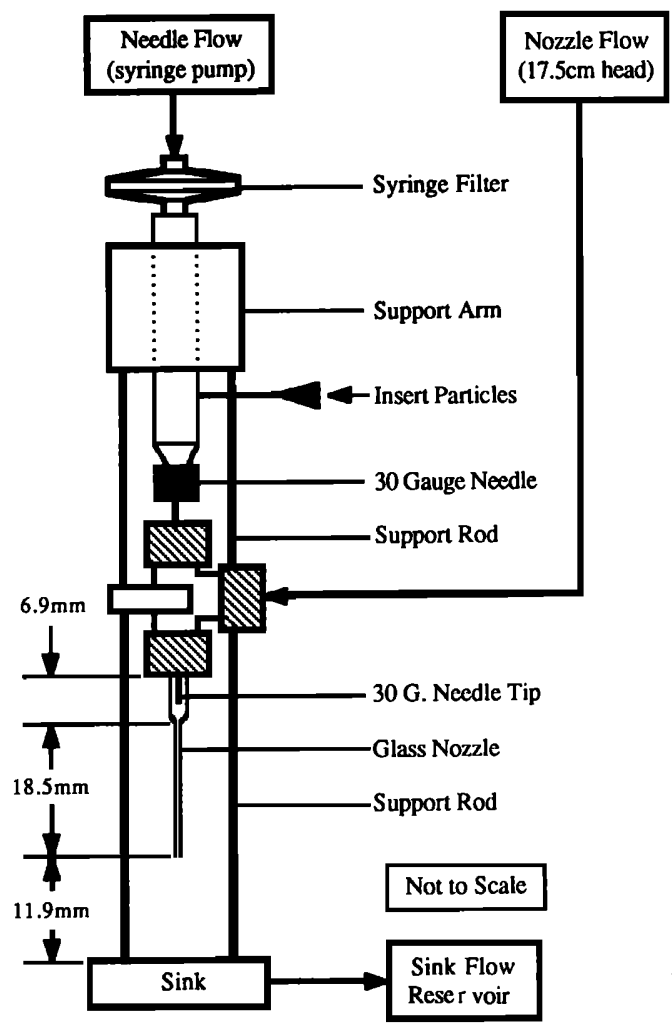

FIG. 3. Coaxial jet-flow assembly used to convect particles through the acoustic confocal region.

transducers. Both transducers and the jet assembly are submerged in the host liquid, which is housed in a Plexiglas tank suspended beneath the mounting plate.

To align the system, we employ a $28-\mu \mathrm{m}$-diam hollow glass sphere adhered to a $12-\mu \mathrm{m}$-diam quartz filament stretched across a Plexiglas bracket. Alignment consists of first positioning the target to maximize the backscattered signal, and then manipulating transducer $B$ to maximize the $90^{\circ}$ echo. The two transducers are thus confocally positioned, with the target situated in the center of the confocal region. The target location is "tagged" using the crosshairs of two filar micrometer microscopes, after which the target is removed and the jet assembly put in place. Using ink to visualize the particle-carrying portion of the flow, the jet assembly is positioned so that the particle flow streamlines pass through the confocal zone. This procedure is repeated prior to every experiment.

Particles studied in this work are convected through the confocal region by the jet flow arrangement illustrated in Fig. 3. This system serves three purposes: (a) to constrain the particle path to a very fine stream that can be precisely positioned; (b) to control the average number of particle events per second; (c) to regulate the particle convection velocity through the confocal region, which in turn determines the number of scattering events per particle event.

Particles enter the jet via a needle positioned slightly upstream of the nozzle contraction. The subsequent convergence of the needle flow streamlines results in an extremely fine stream of particles down the center of the jet proper (nozzle flow). The glass nozzle, which was drawn from stock 3-mm tubing, has an inside diameter at the exit of approximately $270 \mu \mathrm{m}$ with a wall thickness of $100 \mu \mathrm{m}$. A $1-\ell$ glass reservoir provides $17.5 \mathrm{~cm}$ of head, which yields a nozzle flow rate of about $0.017 \mathrm{~cm}^{3} / \mathrm{s}$. The nozzle is approximately cylindrical over a distance of at least 50 diameters, resulting in a fully developed Poiseuille flow. The acoustic confocal region is located $0.37 \mathrm{~cm}$ downstream from the nozzle tip. For a pure water jet, the Reynolds number in the confocal region is about 100 based on the observed jet diameter $(\approx 400 \mu \mathrm{m})$ and centerline flow velocity $(\approx 24 \mathrm{~cm} / \mathrm{s})$. A sink collects the jet far downstream of the confocal region and deposits it into a reservoir on the floor.

Suspended particles are injected into the needle flow at a point far upstream from the hub of the $\mathbf{3 0}$ gauge stainless steel needle (i.d. $=150 \mu \mathrm{m}$ ). The needle flow, which is driven by a syringe pump and passes through a $0.2-\mu \mathrm{m}$ syringe filter, has a flow rate that is typically $0.0002 \mathrm{~cm}^{3} / \mathrm{s}$. By injecting water-soluble ink in place of particles, we can easily visualize the needle flow streamlines. These appear laminar along the entire length of the jet and have a diameter of about $40 \mu \mathrm{m}$ in the plane of the transducers.

The choice of nozzle and needle flow rate is determined by experimentation. To estimate the amplitude of the scattered signal, we must ensure that a sufficient number of scattering events are produced per particle event. We select flow rates that yield the fastest jet possible, while ensuring at least ten scattering events per particle given a pulse repetition frequency (PRF) of $4 \mathrm{kHz}$. Acoustic scattering from the jet is negligible since the same liquid is used for both the jet and the host.

\section{B. Signal generation and detection}

Figure 4 illustrates the instrumentation used to generate the transmitted tone bursts as well as the sinusoids $\cos \omega \mathrm{t}$ and $\sin \omega t$, which are employed by the if demodulator described below. The 2- $\mu$ s-long, 20-mA current pulse activates the double balanced mixers (DBMs) that amplitude modu-

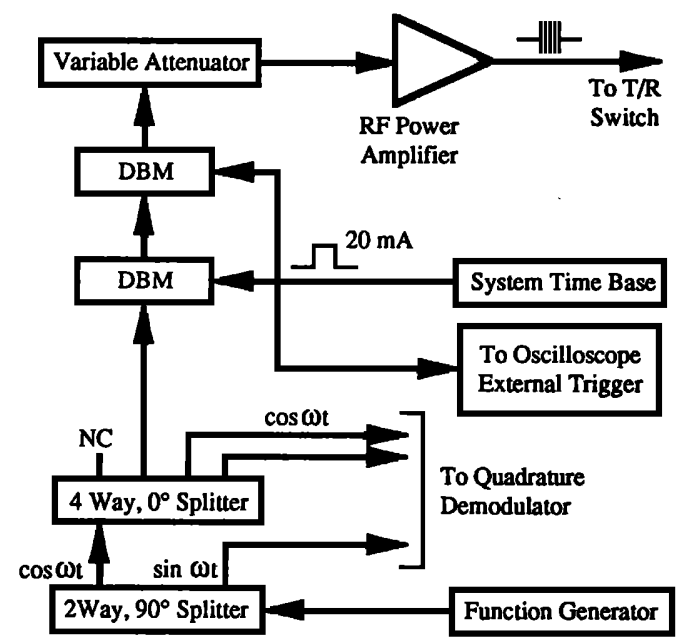

FIG. 4. Block diagram of the circuitry that delivers amplified tone bursts to the transmit signal input of the $T / R$ switch. 


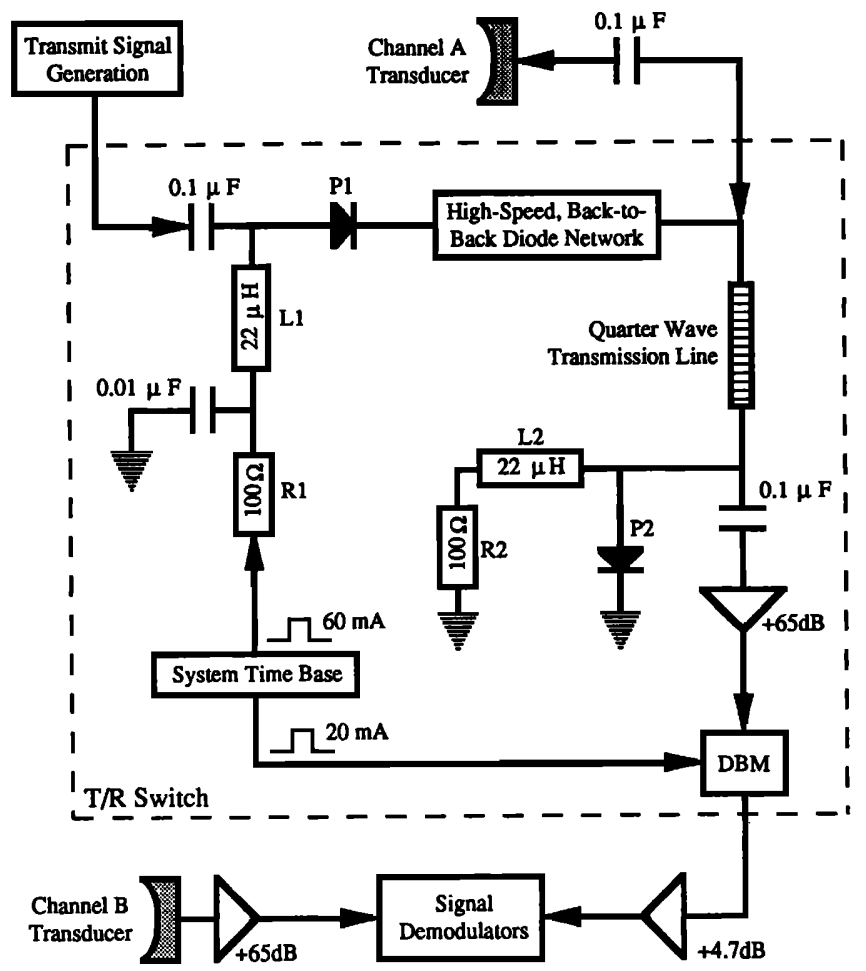

FIG. 5. Block diagram of the $T / R$ switch and the received signal preamplification circuitry.

late the continuous-wave input signal. This results in a $2-\mu \mathrm{s}$ tone burst that is amplified and fed into the transmit signal input of the transmit/receive (T/R) switch.

The $T / R$ switch, which is shown in Fig. 5, is based on a design by Ridpath ${ }^{16}$ and exploits PIN diode and transmission line technology to provide if switching without mechanical relays. PIN diodes are high-speed, current-controlled devices that have typical "on" and "off" resistances of $0.75 \Omega$ and $50 \mathrm{k} \Omega$, respectively.

In the transmit mode both PIN diodes are activated by the 60-mA dc current signal. When turned on, P2 effectively shorts the output of the transmission line, which is reflected back to the transducer side as a high impedance. This results in an rf signal path through $\mathrm{P} 1$ and into the transducer. The transmit mode isolation between the power amplifier and the preamplifier is measured to be $34.3 \mathrm{~dB}$, and the loss from the power amplifier to the transducer is $2.7 \mathrm{~dB}$.

In the receive mode, the current signal is turned off and both $\mathbf{P} 1$ and $\mathbf{P} 2$ cease to conduct, which isolates the transducer from the power amplifier and provides an rf signal path leading from the output of the receiving transducer, through the transmission line, and into the preamplifier. The receive mode loss from the transducer to the preamp is 1.2 dB. Back-to-back diode pairs help reduce broadband amplifier noise that leaks past P1. Received transducer signal amplitudes are on the order of microvolts: thus very small noise levels can corrupt the signal.

Also shown in Fig. 5 is the circuitry used to preamplify

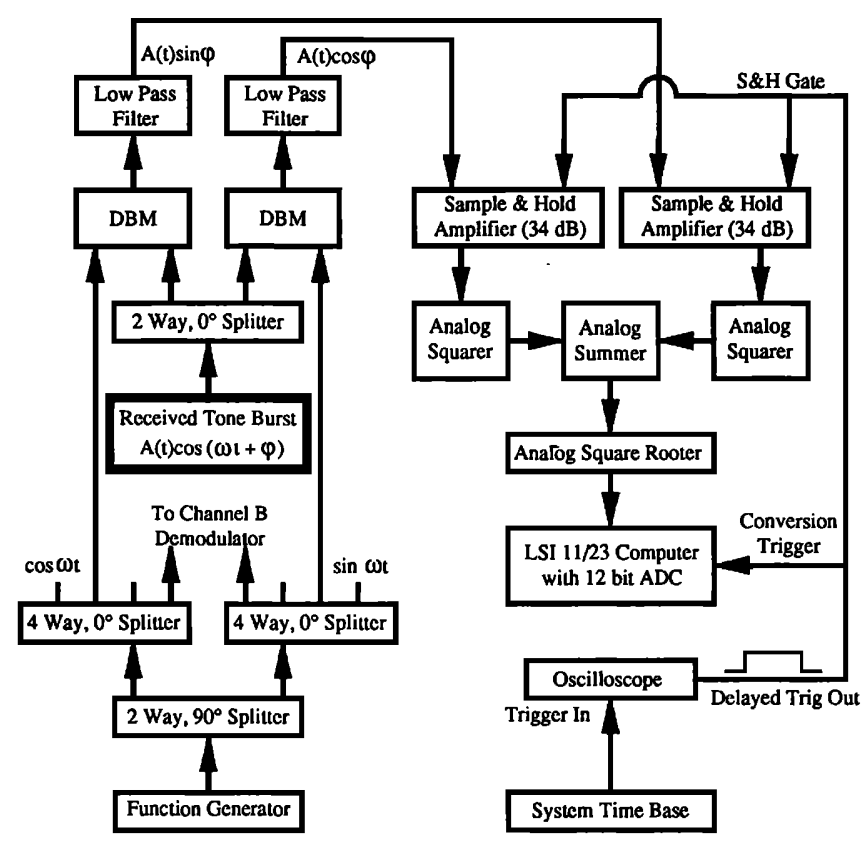

FIG. 6. Block diagram of the quadrature demodulator and signal reconstruction circuitry. Only the channel A system is shown (the channel B configuration is identical).

the rf signals from each detection channel. The channel A system utilizes a DBM configured as a current controlled attenuator, which serves as a backscattered signal gate. This gate is required to keep the main bang leakage through the $T / R$ switch from saturating the channel A demodulator. In order to recover the 3.5-dB insertion loss through the DBM as well as the 1.2-dB loss through the $T / R$ switch, we include an additional $4.7 \mathrm{~dB}$ of gain. This yields an approximate gain balance between the two channels.

\section{Analog signal processing and computer interface}

The task is to determine the amplitude of a scattered tone burst without any information regarding its phase. We employ a quadrature detection scheme, in which the scattered tone burst is treated as an rf carrier signal modulated by a low-frequency envelope function, $\xi(t) \cos (\omega t+\varphi)$, where $\varphi$ is the unknown phase of the received signal. The uncertainty of the phase is a consequence of the uncertainty in the particle position; it is impractical to constrain the particle trajectory well enough to specify the phase to within a fraction of $2 \pi \mathrm{rad}$. We ultimately seek the amplitude of the envelope function, $\xi=|\xi(t)|$, which is proportional to the scattering strength, $\Phi$.

Figure 6 shows a block diagram of the quadrature demodulator. Since each channel utilizes identical demodulators, only one is described here. The signal from the preamplifier stage is first split and then mixed with the sinusoids $\cos \omega \mathrm{t}$ and $\sin \omega \mathrm{t}$. The resulting signals, $\xi(t)[\cos$ $(2 \omega t+\varphi)+\cos (\varphi)]$ and $\xi(t)\left\{\cos \left[2 \omega t+\left(\varphi+90^{\circ}\right)\right]\right.$ $+\sin (\varphi)\}$, are fed into a pair of linear phase, $500-\mathrm{kHz}$ lowpass filters. This yields the detected quadrature components, 
$\xi(t) \cos (\varphi)$ and $\xi(t) \sin (\varphi)$. Using sample and hold amplifiers, we peak detect the quadrature components, producing the dc signals $\xi \cos (\varphi)$ and $\xi \sin (\varphi)$. We then square these signals, sum the products, and take the square root of the sum. The resulting scattered signal amplitude $\xi$ is digitized into a 12-bit word and stored in computer memory. A pair of analog-to-digital conversions (first channel $A$, then channel B) is triggered by the oscilloscope delayed-trigger output pulse.

Data are taken in 2500-point blocks, which corresponds to $0.625 \mathrm{~s}$ of data at a PRF of $4.0 \mathrm{kHz}$. Also obtained are statistics regarding the dc offsets and noise variances for each channel. These values are computed by taking 512 data points while the jet is running without particles while assuming a stationary noise process. The data acquisition program then calculates the means and variances for both channels and writes these statistics into the header for each data file.

The program also offers a "preview mode," in which a 512-point data stream is displayed on a graphics screen, erased, and rewritten with a fresh stream. This process continues until the operator decides to commence recording data. This feature helps reduce the detection of multiple particle events, which result when the particle concentration is too high, a condition which exists immediately after injection of particles into the needle flow. Each experiment involves the acquisition of at least ten blocks of data, which typically correspond to about 1000 particle events.

\section{Digital signal processing}

When a receiver detects a scattered tone burst, it produces an electrical signal. In the absence of noise, the amplitude of the received signal is given by

$$
V_{r}=V_{t} G_{T}(z) G_{R}(z) \Phi,
$$

where $V_{r}$ is the received voltage amplitude, $V_{t}$ is the transmit voltage amplitude, and $G_{T}$ and $G_{R}$ are the gain functions for the transmitting and receiving channels, respectively. The $z$ axis passes through the center of the confocal region and is normal to the plane of the transducers (at which point $z=0$ ). In writing Eq. ( 8 ) we assume that particle trajectories do not deviate significantly from this path. The gain functions account for transducer focusing, electromechanical sensitivity, attenuation in the host liquid, and the gain of the preamplification and analog signal processing circuitry.

As the particle traverses the confocal region, it produces a series of echoes. The $i$ th detected echo can be represented as a tone burst summed with zero-mean, white Gaussian noise. This is written as

$$
s_{i}+n_{i}=\left(V_{r}\right)_{i} e\left(t-\tau_{i}\right) \cos \left(\omega t+\varphi_{i}\right)+n_{i},
$$

where $e\left(t-\tau_{i}\right)$ is a rectangular tone burst envelope (of unit amplitude and duration $T), \tau_{i}$ is the sample time, and $\omega$ is the tone burst center frequency, all of which we know $a$ priori. The $\varphi_{i}$ represent random phase shifts that result from slight deviations in the particle path. Since we are probing the particle at discrete time intervals, the spatial coordinate of the scatterer is treated as a discrete variable given by $z_{i}=\tau_{i} u$, where $u$ is the particle convection velocity in the confocal region. Combining Eqs. (8) and (9) yields

$$
s_{i}+n_{i}=A f_{i} e\left(t-\tau_{i}\right) \cos \left(\omega t+\varphi_{i}\right)+n_{i} .
$$

The sequence $f_{i}=f\left(z_{i}\right)$, which we term the "combined transducer beam profile," represents the normalized spatial dependence of the product of the gain functions and is assumed to be the same for all particle events. Contained within the $f_{i}$ is all the information concerning the transducer beamwidths, the pulse repetition frequency, and the particle convection velocity. The quantity $A$ is the amplitude of the largest echo generated in the course of a particle event. This maximum signal condition occurs as the particle crosses the plane of the transducers. We call $A$ the event amplitude. It is given in terms of the on-axis gain functions, the transmit signal voltage, and the scattering strength by

$$
A=V_{t} G_{T}(0) G_{R}(0) \Phi=G \Phi,
$$

where $G$ is termed the detection channel gain. The scattering strength (and therefore the particle properties) can be inferred from $A$, once we have determined the channel gain through calibration.

For a weakly scattering particle, the precise determination of $A$ is complicated by the presence of noise. Roos derived a maximum likelihood estimator for the event amplitude $^{8}$

$$
A_{m}=\frac{\Sigma f_{i} \xi_{i}}{\Sigma f_{i}^{2}(T / 2)}-\frac{\Sigma N_{0} / 2}{2 \Sigma f_{i} \xi_{i}},
$$

where $N_{0} / 2$ is the variance of the noise and $\xi_{i}$ is the quadrature detected amplitude of the $i$ th scattered tone burst. To implement the estimator, we take the sequence of $\xi_{i}$ that make up the recorded data stream and compute the amplitude estimate at each time step using Eq. (12). Each particle event is represented by a single peak in the resulting sequence of $A_{m}$. The maximum value for a given peak $A_{p}$ is the maximum likelihood estimate for the corresponding event amplitude $A$. Note that we require a priori knowledge of the noise variances and the combined transducer beam profiles for each channel. We determine $N_{0} / 2$ prior to running an experiment by measuring the scattered signal in the absence of particles and, using a technique described in Ref. 8, we obtain the $f_{i}$ by measuring the scattering from a highly monodisperse population of strong scatterers.

\section{E. Determination of particle properties}

In order to characterize a population of unknown particles, we must first calibrate by running known particles and computing the mean value of the measured $A_{p}$. Using an appropriate scattering theory and the calibration particle properties, we then compute the mean scattering strength $\langle\Phi\rangle$. This results in the following estimate for the channel gain:

$$
G_{\theta}=\left\langle A_{\theta}\right\rangle /\left\langle\Phi_{\theta}\right\rangle,
$$

where $\theta$ denotes the scattering angle for the detection channel under consideration (we drop the subscript $p$ for clarity).

We now have sufficient $a$ priori information to calculate the properties of a population of particles provided we possess a relevant and invertible scattering theory in addition to knowledge of the host fluid properties and the particle size 
statistics. Consider, for example, a population of Rayleigh scatterers of known mean volume. By combining Eqs. (7a) and (13), we can express the mean compressibility and density contrasts in terms of the mean event amplitudes, the channel gains, and the mean particle volume, giving

$$
\langle\delta \kappa\rangle=4 \pi\left(\left\langle A_{90}\right\rangle / G_{90}\right) /\left(k^{2}\langle V\rangle\right)
$$

and

$$
\langle\delta \rho\rangle=4 \pi\left[\left(\left\langle A_{90}\right\rangle / G_{90}\right)-\left(\left\langle A_{180}\right\rangle / G_{180}\right)\right] /\left(k^{2}\langle V\rangle\right),
$$

which can be combined with $\mathrm{Eq}$. (7b) to yield the mean particle properties. In writing Eq. (14) we assumed that $\langle\delta \kappa\rangle \propto\langle V\rangle^{-1}$ as opposed to $\left\langle V^{-1}\right\rangle$. This is a good approximation provided the particles are not broadly distributed in volume.

\section{F. Calibration particles}

Effective implementation of our particle characterization technique requires precise and accurate calibration. In addition to being well characterized, ideal calibration particles should be spherical, monodisperse in size, chemically and osmotically stable, easy to handle and store, inexpensive, plentiful, and should last forever.

A possible candidate is the human red blood cell. Much attention has been devoted to the study of the mechanical properties of these cells, ${ }^{2-4}$ and these properties exhibit uniformity within a donor sample as well as from one healthy donor to the next. The mean cellular volume is easily measured using automated cell sizers or bulk methods. Typical values for mean cellular volume, compressibility, and density are $95 \mu \mathrm{m}^{3}$ (which corresponds to a $5.66-\mu \mathrm{m}$-diam sphere), $3.42 \times 10^{-11} \mathrm{~cm}^{2} / \mathrm{dyn}$, and $1.099 \mathrm{~g} / \mathrm{cm}^{3}$, respectively. However, there are numerous drawbacks to using red blood cells as calibration particles. They are nonspherical, their properties change with age, they exist in a limited range of sizes, and they are chemically and osmotically sensitive.

Polystyrene divinylbenzene (PDVB) microspheres provide us with another choice of calibration particle. These spheres are relatively monodisperse, come in a variety of mean sizes, and are chemically stable and osmotically insensitive. The manufacturer provides size and density information, which can also be measured directly using standard techniques. Using a density-gradient method, ${ }^{9}$ Roos found the density of PDVB spheres to be $1.049 \mathrm{~g} / \mathrm{cm}^{3}$, which is in close agreement with the manufacturer's data for both PDVB and polystyrene spheres $\left(1.05 \mathrm{~g} / \mathrm{cm}^{3}\right)$, and with bulk polystyrene density data reported in the literature $(1.05 \mathrm{~g} /$ $\left.\mathrm{cm}^{3}\right)^{14}$

Determining the elastic properties of PDVB spheres is another matter. Compressional and shear wave velocities for bulk polystyrene can be found in the literature. ${ }^{13,17,18}$ Using the reported values, $C=2.380 \times 10^{5} \mathrm{~cm} / \mathrm{s}$ and $c_{s}=1.100 \times 10^{5} \mathrm{~cm} / \mathrm{s}$, along with the published polystyrene density data, we compute [using Eqs. (3) and (6)] the compressibility of bulk polystyrene to be $2.35 \times 10^{-11} \mathrm{~cm}^{2} / \mathrm{dyn}$. Roos measured the acoustic scattering from $5-\mu \mathrm{m}$-diam PDVB spheres in isotonic saline ( $k a=0.31$, where $a$ is the radius of the sphere), after having calibrated the apparatus with human red blood cells. ${ }^{9}$ Using the Rayleigh model, he computed a mean PDVB compressibility of $2.34 \times 10^{-11}$ $\mathrm{cm}^{2} /$ dyn, which is in remarkable agreement with the value for bulk polystyrene. This provides justification for adopting the published compressional and shear wavespeeds for bulk polystyrene as characteristic of PDVB spheres as well.

\section{EXPERIMENTAL PROCEDURE}

\section{A. Experiments with PDVB microspheres}

To test this hypothesis we measured the scattering from individual suspensions of monodisperse (mean diameters ranged from 4.9 to $14.6 \mu \mathrm{m}$ ) PDVB spheres. We then computed, for each sample, the mean particle properties as well as a farfield, "relative" differential scattering cross section given by

$$
\sigma_{\theta} \equiv\left|\Phi_{\theta}\right|^{2} /\left(\pi a^{2}\right)=\left(\left\langle A_{\theta}\right\rangle / G_{\theta}\right)^{2} /\left(\pi a^{2}\right) .
$$

Experimental cross sections were then compared to theoretical calculations based on the elastic properties of bulk polystyrene. Since the $4.9-\mu \mathrm{m}$ spheres were previously calibrated against a reliable standard (blood cells), they were used to calibrate the system.

Table I gives the mean diameter for each of the PDVB populations employed, as well as the estimated error and precision of the mean. The manufacturer (Duke Scientific, Palo Alto, CA) sizes a 100-point sample using a light microscope calibrated against an NBS stage micrometer. In addition, the vendor runs the spheres through a Coulter model $Z_{B}$ particle analyzer, which generates diameter and volume statistics for a 10000 -point sample. All diameters given in Table I are the electrically determined values, which we considered to be more precise since the mean is calculated over a much larger sample of the parent population. Stated errors correspond to the differences between the optically and electrically determined means. As a measure of precision, we used the standard deviation computed by the particle analyzer.

Listed in Table II are the estimated mechanical properties for the PDVB spheres, using the polystyrene data reported in the literature. The stated PDVB density error represents the disagreement among the published values. Error in the compressibility corresponds to the difference between the measurements performed by Roos, and the computation using the published polystyrene data. We lack sufficient information to infer the error associated with the wave speeds. Also listed in Table II are the properties of distilled, degassed water that we used as a host fluid. The density and sound velocity (from which we obtain the compressibility) were

TABLE I. Mean sizes for each PDVB suspension used in this study along with estimates of precision and error.

\begin{tabular}{cc}
\hline \hline $\begin{array}{c}\text { Mean diameter } \\
(\mu \mathrm{m})\end{array}$ & $\begin{array}{c}\text { Estimated error } \\
(\%)\end{array}$ \\
\hline $4.9 \pm 0.5$ & \pm 3.0 \\
$5.9 \pm 0.5$ & \pm 1.0 \\
$7.4 \pm 0.6$ & \pm 6.0 \\
$9.7 \pm 0.2$ & \pm 1.2 \\
$10.5 \pm 0.9$ & \pm 8.6 \\
$14.6 \pm 0.4$ & \pm 0.2 \\
\hline \hline
\end{tabular}


TABLE II. Mechanical properties of PDVB spheres and the host liquid.

\begin{tabular}{lll}
\hline \hline \multicolumn{3}{c}{ PDVB microspheres } \\
\hline Compressibility & $2.34 \times 10^{-11} \mathrm{~cm}^{2} / \mathrm{dyn}$ & $\pm 0.4 \%$ \\
Density & $1.049 \mathrm{~g} / \mathrm{cm}^{3}$ & $\pm 0.1 \%$ \\
Compressional wave speed & $2.380 \times 10^{5} \mathrm{~cm} / \mathrm{s}$ & \\
Shear wave speed & $1.100 \times 10^{5} \mathrm{~cm} / \mathrm{s}$ & \\
\multicolumn{3}{c}{ Distilled water $\left(\right.$ temperature $\left.=23 \pm 2{ }^{\circ} \mathrm{C}\right)$} \\
Compressibility & $4.51 \times 10^{-11} \mathrm{~cm}^{2} / \mathrm{dyn}$ & $\pm 0.7 \%$ \\
Density & $0.9976 \mathrm{~g} / \mathrm{cm}^{3}$ & $\pm 0.07 \%$ \\
\hline
\end{tabular}

measured using a Mettler-Parr DMA 40 digital density meter and a Nusonics 6105 sonic solution monitor. Tabulated errors correspond to measured changes in the host properties that resulted from variations in room temperature, which were typically $23 \pm 2{ }^{\circ} \mathrm{C}$.

When performing an experiment, calibration particles were run first. A dilute PDVB particle suspension was obtained by dispersing one drop of the vendor's suspension into about $0.5 \mathrm{~m} \ell$ of host liquid, and introduced into the apparatus by injecting $\approx 0.1 \mathrm{~m} \ell$ of this into the needle flow. Using the data acquisition program in the preview mode, we monitored the spheres as they crossed the confocal region. When the particle event statistics appeared acceptable (many events with few multiple peaks), data acquisition commenced. Using elastic scattering theory [Eq. (4) ], the measured mean event amplitudes for each channel were combined with the PDVB and host properties to yield the channel gains.

Because of the limited dynamic range of the system, we could not run all particle sizes at the same transmit signal level. We took data with the 4.9-, 5.9-, and 7.4- $\mu$ m-diam spheres at a transmit level of $40 \mathrm{~V}$ peak-to-peak, and then reduced to $15 \mathrm{~V}$ peak-to-peak in order to run the 7.4-, 9.7-, $10.5-$, and $14.6-\mu \mathrm{m}$ particles. Using the $7.4-\mu \mathrm{m}$ results, we were able to normalize the $15-\mathrm{V}$ data to the $40-\mathrm{V}$ data. From the scattering data for each sphere size, we determined the mean event amplitudes and generated histograms of event amplitude statistics. Using the Rayleigh approximation, the mean particle properties were calculated for all of the PDVB samples. Finally, we computed the mean scattering strengths for each sample, from which the scattering cross sections followed using Eqs. (2)-(8).

\section{B. Estimates of precision}

Experimental precision was based on the precision of the calibration particle size estimate and the measured precisions of the mean event amplitudes computed from both the calibration data and the unknown particle data. Every experiment yielded ten blocks of data, each of which accounted for roughly 100 particle events. We computed the sample mean for each block and found that the variation of the sample means was generally equal to about two standard errors computed over all of the events. This is consistent with observations made by Roos, who attributed the discrepancy to gradual fluctuations of the position of the jet. We therefore chose, as did Roos, to estimate the precision of the mean event amplitude by computing the standard deviation of the sample means themselves. This value generally fell between $1.5 \%$ and $4.0 \%$.

When calculating quantities of interest (cross sections, particle properties, etc.) we propagated uncertainties by using a standard perturbation technique, where the relevant equations were expanded in a Taylor series to first order in the perturbed parameters. It was assumed that the fluctuations in the event amplitude measurements at different angles were uncorrelated. The coefficients in the expansion were calculated using the assumed physical parameters (calibration particle and host properties, etc.) and the computed mean event amplitudes.

\section{EXPERIMENTAL RESULTS}

\section{A. Particle properties}

Plotted in Fig. 7 are the theoretical scattering cross sections for PDVB spheres in water versus $k a$ using the Rayleigh approximation as well as the exact solution for elastic scatterers. Also plotted are the cross sections computed using the experimental results, where the error bars denote the precision of the calculation. The close agreement between the measured cross sections and those predicted by the exact
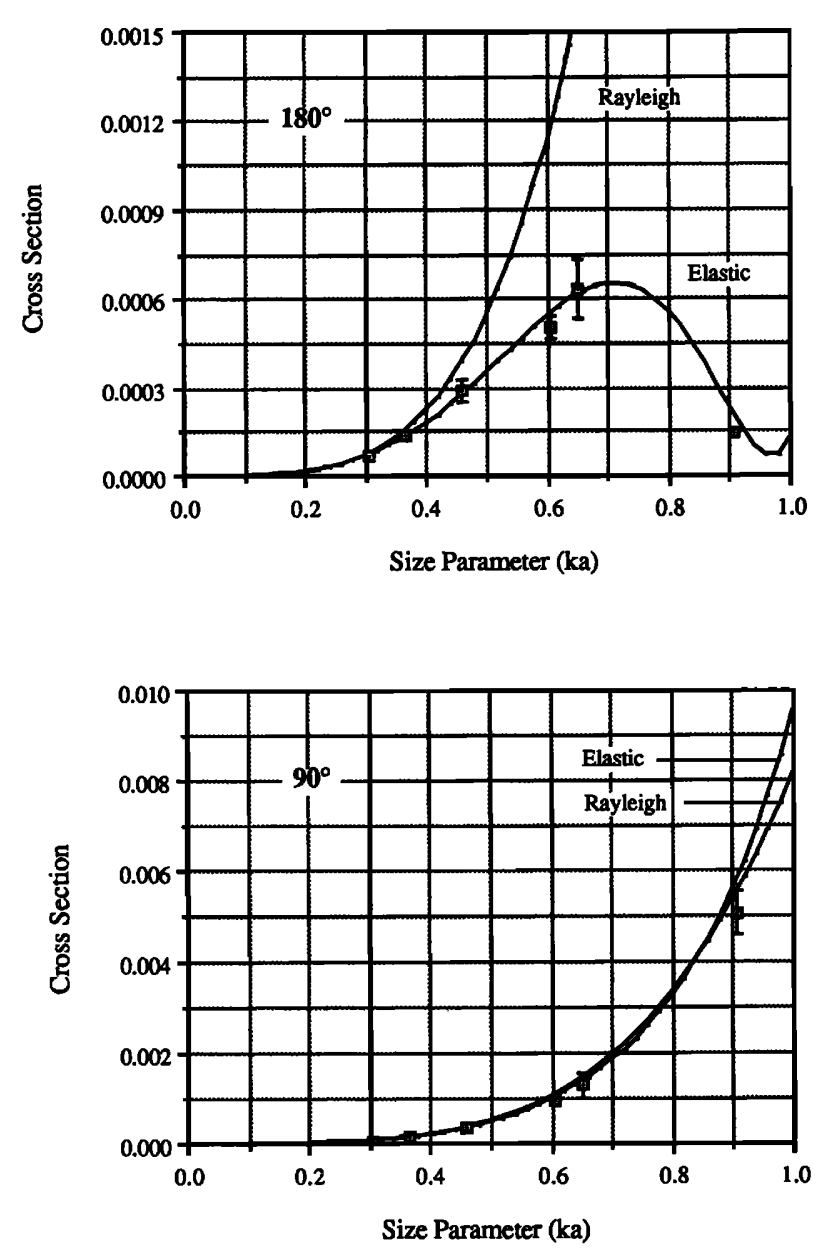

FIG. 7. A comparison of the measured scattering cross sections for PDVB spheres in water with predictions based on elastic scattering theory and the Rayleigh approximation. 
TABLE III. Measured mean compressibility and density for each PDVB suspension. The apparatus was calibrated using the $4.9-\mu \mathrm{m}$-diam spheres using Rayleigh scattering theory.

\begin{tabular}{rcc}
\hline \hline $\begin{array}{c}\text { Diameter } \\
(\mu \mathrm{m})\end{array}$ & $\begin{array}{c}\text { Compressibility } \\
\left(\times 10^{-11} \mathrm{~cm}^{2} / \mathrm{dyn}\right)\end{array}$ & $\begin{array}{c}\text { Density } \\
\left(\mathrm{g} / \mathrm{cm}^{3}\right)\end{array}$ \\
\hline $4.9(k a=0.30)$ & $2.34 \pm 0.09$ & $1.049 \pm 0.027$ \\
$5.9(k a=0.37)$ & $2.33 \pm 0.07$ & $1.031 \pm 0.024$ \\
$7.4(k a=0.46)$ & $2.34 \pm 0.07$ & $0.991 \pm 0.020$ \\
$9.7(k a=0.60)$ & $2.43 \pm 0.06$ & $0.904 \pm 0.015$ \\
$10.5(k a=0.65)$ & $2.44 \pm 0.10$ & $0.895 \pm 0.020$ \\
$14.6(k a=0.90)$ & $2.38 \pm 0.07$ & $0.692 \pm 0.010$ \\
\hline \hline
\end{tabular}

elastic theory suggests that the acoustic scattering apparatus did not bias the measurements in such a way as to yield nonphysical results. Since they are referenced to a $4.9-\mu \mathrm{m}$ calibration particle whose properties we presume to know, the cross-section measurements are not absolute. However, there is good agreement between theory and experiment over the entire range of sizes under study. This self-consistency supports our contention that the values listed in Table II adequately describe the mechanical properties of PDVB spheres.

Table III shows the measured compressibilities and densities for each size sphere using the Rayleigh theory to both calibrate and compute the mean particle properties. The compressibilities all agree with each other to within the stated precision, but the densities exhibit a significant reduction with increasing particle size. This bias, which was observed previously by Roos, ${ }^{9}$ results from the fact that the Rayleigh approximation overestimates the backscattered signal strength for $k a$ larger than about 0.4 . This breakdown in the long-wavelength assumption leads to a reduction in the computed density contrast which, for PDVB spheres in water, corresponds to a decrease in the computed density. Recall that the compressibility calculations are made using only $90^{\circ}$ scattering information, where the Rayleigh and elastic theories show relatively close agreement. This explains the absence of a similar bias in the compressibility values.

The inability of Rayleigh theory to predict the scattering from PDVB spheres larger than about $5 \mu \mathrm{m}$ is of no consequence to the calibration issue. All we require for calibration is a well-characterized population of monodisperse (in size) particles and a solution to the direct scattering problem. The agreement between predictions using the exact elastic theory and measurements performed with PDVB spheres suggests that these spheres can serve as effective calibration particles for sizes up to $k a=1$.

\section{B. Distributions}

Representative distributions of the particle event amplitudes are given in Fig. 8 for 5.9- and 14.6- $\mu$ m-diam microspheres. Note that although their volumes differ by a factor of 15 , the backscattering from these spheres appears very similar. This serves to further illustrate the limitations of the Rayleigh approximation, which predicts a nearly linear relation between the scattered strength and particle volume.
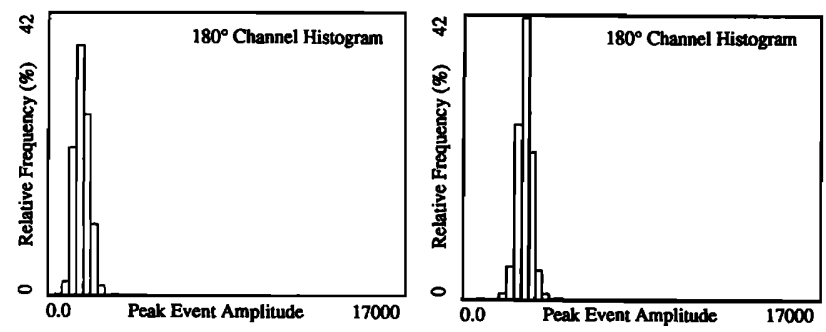

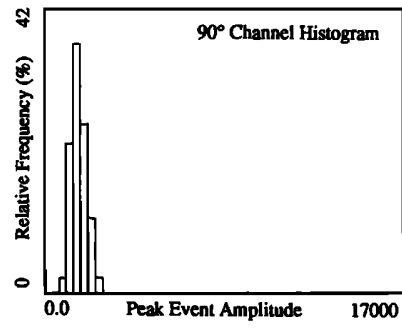

$5.9 \mu \mathrm{m}$ diameter particles

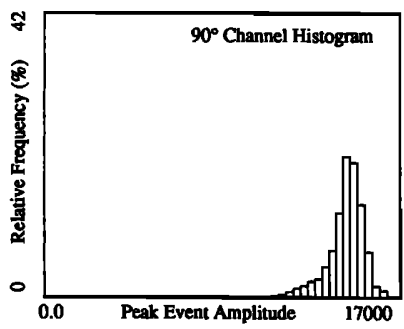

$14.6 \mu \mathrm{m}$ diameter particles
FIG. 8. Distributions of measured event amplitudes for 5.9- and 14.6- $\mu \mathrm{m}$ diam PDVB spheres in water.

However, at $90^{\circ}$ the Rayleigh theory succeeds in predicting PDVB behavior. Thus the $90^{\circ}$-event amplitudes are proportional to the scatterer volume, provided the particles are narrowly distributed in density and compressibility. Consider the sequence of $90^{\circ}$-event histograms given in Fig. 9. These are distributions of particle size computed from the cube root of the measured event amplitude. The ability of the acoustic scattering apparatus to discriminate size is clear from this series of plots. Also evident is the variation in the distributions from one particle size to the next. In Table IV we present the computed standard deviations of the size dis-
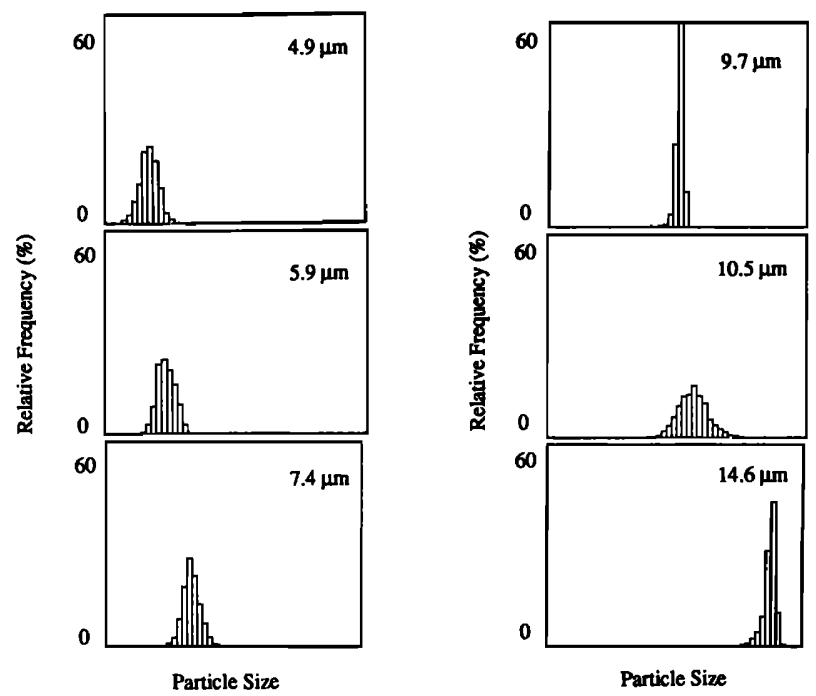

FIG. 9. Channel B $\left(90^{\circ}\right)$ histograms for PDVB spheres in water. The horizontal axis corresponds to the cube root of the event amplitude, which for PDVB is approximately proportional to size. 
TABLE IV. Standard deviations of the measured PDVB distributions for each particle suspension. Also tabulated are the same statistics measured with a Coulter counter (supplied by the manufacturer).

\begin{tabular}{rrrr}
\hline \hline $\begin{array}{c}\text { Diameter Standard deviation } \\
(\mu \mathrm{m})\end{array}$ & $\begin{array}{c}\text { Standard deviation } \\
\text { (channel A) }\end{array}$ & $\begin{array}{c}\text { Standard deviation } \\
\text { (Coulter counter) }\end{array}$ \\
\hline 4.9 & $9.2 \%$ & $9.3 \%$ & $12 \%$ \\
5.9 & $7.1 \%$ & $7.3 \%$ & $8.0 \%$ \\
7.4 & $5.9 \%$ & $6.4 \%$ & $8.1 \%$ \\
9.7 & $3.1 \%$ & $3.0 \%$ & $2.1 \%$ \\
10.5 & $4.4 \%$ & $7.4 \%$ & $8.7 \%$ \\
14.6 & $4.2 \%$ & $3.4 \%$ & $2.7 \%$ \\
\hline \hline
\end{tabular}

tributions for each particle diameter, along with the Coulter analyzer statistics provided by the manufacturer. The statistics for the distributions using the two techniques are comparable. Since the Coulter device is sensitive only to volume changes, this suggests that the compressibility and density of the PDVB spheres are narrowly distributed compared to their size.

\section{CONCLUSIONS}

In this paper we have addressed the problem of quantitative microparticle characterization using scattered ultrasonic tone bursts. This approach is unique in that it brings the advantages of a single-particle observation technique to the problem of density and compressibility measurement. One can either compute mean properties over a population of particles, or display histograms of scattered signal statistics.

Technical problems associated with calibration have been considered (in particular the choice of calibration particle). Although Rayleigh scattering theory proved ineffective at predicting the backscattering cross section of PDVB microspheres, the experimental data exhibit close agreement with Hay and Burling's exact solution for elastic scatterers computed using the measured mechanical properties of bulk polystyrene. Since an invertible scattering theory is not required for calibration, we will make use of the PDVB spheres as reference particles for future experiments.

Because of its sensitivity and particle handling characteristics, the instrument is well suited to in vitro mechanical characterization of biological particles. With the exception of the maximum likelihood estimator (which can be bypassed given high signal-to-noise conditions), all signal processing is performed by analog circuits, which makes the apparatus amenable to real-time applications, such as process monitoring or selective sorting based on mechanical descriptors.

In this paper we present distributions of measured event amplitudes, but not of computed particle properties. This is because independent knowledge of the size of a particle is required in order to determine its compressibility and density. Due to this limitation, it is impossible for us to establish independently the distributions of particle properties in a heterogeneous population. Efforts are under way to incorporate individual particle sizing through the addition of an electrozone sensing orifice upstream of the confocal region. ${ }^{19}$

\section{ACKNOWLEDGMENTS}

We gratefully acknowledge the assistance and advice of Xucai Chen, Chaur Jian Hsu, and Stephen Wardlaw as well as the financial support of the National Institutes of Health, Grant R01-GM30419, and the Office of Naval Research.

'S. H. Chen and M. Holz, "Medical application of photon correlation spectroscopy," Med. Res. Eng. 12, 19-25 (1977).

${ }^{2}$ R. C. Leif, "Buoyant density separation of cells," in Automated Cell Identification and Cell Sorting, edited by G. L. Wied and G. F. Bahr (Academic, New York, 1970), pp. 22-96.

${ }^{3}$ K. K. Shung, B. A. Krisko, and J. O. Ballard, "Acoustic measurement of erythrocyte compressibility," J. Acoust. Soc. Am. 72, 1364-1367 (1982).

${ }^{4}$ M. A. H. Weiser and R. E. Apfel, "Extension of acoustic levitation to include the study of micron-size particles in a more compressible host liquid," J. Acoust. Soc. Am. 71, 1261-1268 (1982).

${ }^{5}$ J. A. Steinkamp, M. J. Fulwyler, J. R. Coulter, R. D. Hiebert, J. L. Horney, and P. F. Mullaney, "A new multiparameter separator for microscopic particles and biological cells," Rev. Sci. Instrum. 44, 1301-1310 (1973).

${ }^{6} \mathrm{D}$. Holve and S. A. Self, "Optical particle sizing for in situ measurements, part 1," Appl. Opt. 18, 1632-1645 (1979).

${ }^{7}$ R. C. Leif, "A proposal for an automatic multiparameter analyzer for cells," in Automated Cell Identification and Cell Sorting, edited by G. L. Wied and G. F. Bahr (Academic, New York, 1970), pp. 131-159.

${ }^{8} \mathrm{M}$. S. Roos, "A technique for the study of acoustic scattering from microparticles" J. Acoust. Soc. Am. 83, 770-776 (1988).

${ }^{9}$ M. S. Roos, R. E. Apfel, and S. C. Wardlaw, "Application of $30-\mathrm{MHz}$ acoustic scattering to the study of human red blood cells," J. Acoust. Soc. Am. 83, 1639-1644 (1988).

${ }^{10} \mathrm{~V}$. C. Anderson, "Sound scattering from a fluid sphere," J. Acoust. Soc. Am. 22, 426-431 (1950).

"J. J. Faran, "Sound scattering by solid cylinders and spheres," J. Acoust. Soc. Am. 23, 405-418 (1951).

'2R. Hickling, "Analysis of echoes from a solid elastic sphere in water," J. Acoust. Soc. Am. 34, 1582-1592 (1962).

${ }^{13}$ A. E. Hay and R. W. Burling, "On sound scattering and attenuation in suspensions, with marine applications," J. Acoust. Soc. Am. 72, 950-959 (1982).

${ }^{14}$ Lord Rayleigh, The Theory of Sound, Second Edition, Volume II (Dover, New York, 1945), p. 282.

${ }^{15}$ R. A. Roy, "Quantitative particle characterization by scattered ultrasound," Ph.D. thesis, Yale University, 1987; University Microfilms, Ann Arbor, MI.

${ }^{16}$ I. Ridpath, "T-R Switching with PIN Diodes," QST 65(3), 19-21 (1981).

${ }^{17}$ J. R. Allegra and S. A. Hawley, "Attenuation of sound in suspensions and emulsions: Theory and experiment," J. Acoust. Soc. Am. 51, 1545-1564 (1972).

${ }^{18} \mathrm{R}$. Kono, "The dynamic bulk viscosity of polystyrene and polymethyl methacrylate," J. Phys. Soc. Jpn. 15, 718-725 (1959).

${ }^{19} \mathrm{X}$. Chen, R. A. Roy, and R. E. Apfel, "Advances in microparticle characterization using high-frequency ( $30-\mathrm{MHz}$ ) acoustic scattering, J. Acoust. Soc. Am. Suppl. 184, S163 (1988). 